\title{
Erratum to: Effects of tolvaptan in the early postoperative stage after heart valve surgery: results of the STAR (Study of Tolvaptan for fluid retention AfteR valve surgery) trial
}

Hiroyuki Nishi $^{1} \cdot$ Koichi Toda $^{1} \cdot$ Shigeru Miyagawa $^{1} \cdot$ Yasushi Yoshikawa $^{1}$.

Satsuki Fukushima ${ }^{1} \cdot$ Masashi Kawamura $^{1} \cdot$ Daisuke Yoshioka $^{1} \cdot$ Tetsuya Saito $^{1}$.

Takayoshi Ueno $^{1} \cdot$ Toru Kuratani $^{1} \cdot$ Yoshiki Sawa $^{1}$

Published online: 30 October 2015

(C) Springer Japan 2015

\section{Erratum to: Surg Today}

DOI 10.1007/s00595-015-1251-y

Throughout this article, "agonist" should read "antagonist".

The online version of the original article can be found under doi:10.1007/s00595-015-1251-y.

Yoshiki Sawa

sawa-p@surg1.med.osaka-u.ac.jp; nishi24@jc4.so-net.ne.jp

1 Department of Cardiovascular Surgery, Osaka University Graduate School of Medicine, 2-2, Yamada-Oka, Suita, Osaka 565-0871, Japan 